\title{
IMPACTO DA PANDEMIA DE COVID-19 NO AGRONEGÓCIO BRASILEIRO
}

\author{
Maiara Cristina Metzdorf Silva, Jessica Marciella Almeida Rodrigues, Oscar Mitsuo Yamashita \\ Universidade do Estado de Mato Grosso - UNEMAT, Alta Floresta, MT. E-mail: metzdorf mt@hotmail.com
}

\section{RESUMO}

Em 2020 o mundo mudou, vivenciando um novo cenário, decorrente da pandemia do coronavírus, que se espalhou rapidamente, causando inúmeras vítimas. Essa situação que ainda permanece, provocou uma tragédia de proporções épicas e, devido ao despreparo, especialmente por parte dos profissionais da área da saúde, houve medidas extremas, como isolamento social e o fechamento das fronteiras em uma tentativa desesperada de conter a disseminação da doença. Devido a este cenário, muitos setores empresariais foram prejudicados, amargando prejuízos e desafios, provocados pela pandemia que se alastrou rapidamente por todo mundo. O presente trabalho teve como objetivo apresentar os desafios e impactos vivenciados pelo agronegócio brasileiro, neste cenário de pandemia do coronavírus. $\mathrm{O}$ estudo foi realizado por meio de pesquisa bibliográfica baseada em artigos, jornais, revistas, cobertura de textos postados na internet e outros meios de comunicação virtual de ampla e rápida disseminação, que abordam a pandemia de Covid-19 e seus efeitos neste setor que ajuda a sustentar a economia brasileira. Estudos confirmam que o agronegócio no Brasil mais uma vez se mostrou um setor forte, apresentando bons resultados em meio a um período de retração econômica e inúmeros desafios diante dos impactos da pandemia.

Palavras-chave: Coronavírus. Prejuízos. Cadeia produtiva.

\section{IMPACT OF THE COVID-19 PANDEMIC ON BRAZILIAN AGRIBUSINESS}

\begin{abstract}
In 2020, the world changed, experiencing a new scenario, resulting from the coronavirus pandemic, which spread rapidly, causing countless victims. This situation that still remains, caused a tragedy of epic proportions and, due to lack of preparation, especially on the part of health professionals, there were extreme measures, such as social isolation and the closing of borders in a desperate attempt to contain the spread of the disease. Due to this scenario, many business sectors were harmed, suffering losses and challenges, caused by the pandemic that spread quickly throughout the world. The present work aimed to present the challenges and impacts experienced by the Brazilian agribusiness in this coronavirus pandemic scenario. The study was carried out through bibliographical research based on articles, newspapers, magazines, coverage of texts posted on the internet and other wide and rapid dissemination means of virtual communication, which address the Covid-19 pandemic and its effects on this helping sector to sustain the Brazilian economy. Studies confirm that agribusiness in Brazil has once again proved to be a strong sector, showing good results amidst a period of economic downturn and numerous challenges in the face of the impacts of the pandemic.
\end{abstract}

Keywords: Coronavirus. Losses. Productive chain. 


\section{INTRODUÇÃO}

O ano de 2020 ficará marcado na história da humanidade devido à pandemia do novo coronavírus. Com o epicentro na província de Hubei, na China, esta doença desconhecida, associada à pneumonia, foi posteriormente denominada COVID-19.

Os coronavírus pertencem à família Coronaviridae, sendo composto pelos gêneros Alphacoronavirus e Betacoronavirus, que geralmente infectam mamíferos e aves (DUARTE, 2020). O surto ocasionado por um novo coronavírus é caracterizado por evoluir para pneumonia, síndrome respiratória aguda (ZHU et al., 2019). Estudos apontam como foco dos primeiros casos de COVID-19 o mercado de frutos do mar de Huanan (QUN Ll et al., 2020). E, diante deste cenário, o mundo testemunha a acelerada disseminação dos casos da síndrome respiratória aguda grave (LEIVA, 2020).

$\mathrm{Na}$ ausência de medicamentos e métodos que contenham o COVID-19, a Organização Mundial da Saúde (OMS) definiu o distanciamento como melhor método para reduzir a velocidade de contágio (LAU et al., 2020).

Até 21 de junho de 2021, houve 178.202.610 casos confirmados de COVID-19, incluindo 3.865.738 mortes, notificados à OMS. Com uma rápida e devastadora propagação do novo coronavírus no mundo, a Organização Mundial de Saúde (OMS) decretou estado de pandemia global, adotando medidas rígidas de isolamento social. Neste cenário, a maioria dos setores da economia como o comércio, a indústria, o turismo e o agronegócio foram afetados. E isso ocasionou o pior desempenho para a economia mundial no ano de 2020 (SOENDERGAARD et al., 2020).

Esta "nova" situação impactou tanto os processos de produção quanto às transações comerciais rotineiras. E no agronegócio, não foi diferente, causando efeitos em todos os níveis dos processos produtivos.

O objetivo deste artigo de revisão é apresentar e discutir os desafios e impactos vivenciados pelo setor do agronegócio brasileiro, neste cenário da pandemia pelo coronavírus.

\section{MÉTODOS}

A metodologia centrou-se no seguinte eixo de operacionalização: organização de uma pesquisa bibliográfica baseada em artigos, jornais, revistas, abrangendo textos postados na internet, e outros meios de comunicação virtual de amplo alcance e rápida disseminação, que abordam a pandemia de COVID-19 e seu efeito no agronegócio brasileiro. Foram utilizados dados disponíveis nas plataformas eletrônicas Scielo, Google Acadêmico, Periódicos CAPES/MEC. Para a presente busca, foram incluídos artigos completos de língua inglesa e portuguesa, publicados especialmente no ano de 2020. O estudo desenvolvido neste artigo, sinaliza a continuidade, já que a pandemia está em rápida progressão e muitas informações são dinâmicas, com alterações em curtos intervalos de tempo.

\section{RESULTADOS E DISCUSSÃO}

$O$ agronegócio envolve atividades que ocorrem em toda a cadeia produtiva (CUNHA; ESPÍNDOLA, 2015). Corresponde a todas as atividades econômicas relacionadas com a produção, apresentando grande importância para a economia global e é um dos pilares econômicos do Brasil (HEREDIA; PALMEIRA; LEITE, 2010).

$O$ agronegócio funciona como um setor econômico, dada a sua importância para economia e a sociedade. Isto se deve ao envolvimento entre cadeia de produção alimentar e a sua íntima interação com os mais variados setores, como a agricultura, pecuária, indústria e prestação de serviços (TALIARINE; RAMOS; FAVORETTO, 2015).

Nas duas últimas décadas, o desenvolvimento do agronegócio brasileiro, promoveu uma explosão no crescimento, impulsionado pelo aumento vertiginoso da produção, permitindo que o Brasil se firmasse como um dos mais importantes fornecedores de alimentos para o mundo (MAURI et al., 2017). O aumento das produções contribuíram para a redução dos preços dos alimentos, colaborando com a melhoria da qualidade de vida e saúde da população, com maior poder de compra (NEVES et al., 2019).

O agronegócio é reconhecido como condutor importante para o crescimento econômico brasileiro. As somas de bens gerados no agronegócio chegaram a $\mathrm{R} \$ 1,55$ trilhão, ou $21,4 \%$ do PIB brasileiro (BARROS; CASTRO, 2020). Dentre os segmentos, a maior parcela é do ramo agrícola, que corresponde a $68 \%$ desse valor (R\$ 1,06 trilhão) e a pecuária corresponde a $32 \%$, ou seja, $\mathrm{R} \$ 494,8$ bilhões (CNA, 2020).

Quanto à exportação brasileira, em 2019, $43 \%$ do total exportado foram de produtos do agronegócio, demonstrando sua importância e 
contribuição para o crescimento da economia brasileira. O Brasil é o maior exportador de açúcar, café, suco de laranja, soja em grãos, carnes bovina, frango e o terceiro maior de milho, e o quarto de carne suína (CNA, 2020).

O Brasil está na quarta posição de maior exportador mundial de produtos agropecuários, atrás apenas dos EUA, China e União Europeia. E esse crescimento contribuiu significativamente para a estabilidade macroeconômica do Brasil (NONNENBERG, 2010).

Contudo, em 2020, o surgimento de uma nova doença, denominada COVID-19, abalou as estruturas de diversos setores econômicos, incluindo o agronegócio. A primeira ocorrência foi notificada pela China, para a Organização Mundial de Saúde (OMS), que emitiu o primeiro comunicado em 31 de dezembro de 2019. A primeira onda atingiu a China entre janeiro e fevereiro de 2020, e em seguida, a segunda onda da doença gerou uma proliferação gradativa pelo resto do planeta (SOENDERGAARD et al., 2020).

Em 11 de março de 2020, a OMS emitiu um alerta, designando a doença como pandemia global, gerando colapsos sucessivos nos sistemas de saúde dos países com maior taxa de infectados (OMS, 2020). Diante do cenário, com a aceleração na contaminação do COVID-19, houve o fechamento das fronteiras, medida recomendada pela OMS, na tentativa de reduzir as infecções.

Essa situação fez com que diversos setores suspendessem suas atividades, gerando impactos econômicos negativos, decorrentes da crise do novo coronavírus, relacionados ao isolamento social, reduzindo assim, a produção e aumentando o consumo.

O efeito do isolamento atingiu de imediato o escoamento de insumos e a produção agrícola brasileira, causando problemas logísticos e a perda de produção (SOENDERGAARD et al., 2020).

Quando o Brasil alcançou um elevado número de infectados, iniciou-se a restrição da entrada de contêineres brasileiros que chegavam na China e outros vários países, impedindo o desembarque dos produtos brasileiros naquele país, contribuindo para a queda da demanda em diferentes setores, incluindo os frigoríficos e, com isso, provocando a suspensão nas operações de exportação (BARCELLOS; REUPKE, 2020).

Segundo a Editora Globo (2020), no final de 2020, autoridades sanitárias da cidade de Jinan, na China, detectaram a presença de coronavírus em bifes e tripas bovinas e nas embalagens dos produtos brasileiros. Segundo as informações desta mesma fonte, os produtos entraram pelos portos de Xangai. Dessa maneira, a China aumentou o rigor e o número de testes em alimentos congelados oriundos do Brasil, após confirmar a presença do vírus nos produtos importados. Apesar disso, a OMS destaca que o risco de transmissão do novo coronavírus em alimentos congelados seria muito baixo.

Diante deste cenário, as dificuldades e desafios se tornaram mais acentuados, provocando o fechamento temporário de importantes setores ligados direta ou indiretamente ao agronegócio, como os frigoríficos e a paralisação de empresas de transportes, provocando problemas de escoamento dos produtos e alimentos (CARDOSO; COSTA, 2020). O fechamento das empresas que fornecem serviços de manutenção a frota de veículos de transporte rodoviário foi outro fator que também interferiu na logística e na distribuição dos produtos brasileiros (GUERRA, 2020).

Os efeitos provocados pelas restrições nos setores primários do agronegócio também causaram reflexos nos demais segmentos da economia, sendo rapidamente verificadas quedas na demanda, na distribuição e no consumo (GUASQUE; GUASQUE, 2020) e, devido ao fechamento obrigatório dos setores de prestação de serviços como restaurantes, feiras livres e comércios, que consumiam grande parcela de produtos alimentícios produzidos pelo agronegócio, a situação tornou-se economicamente grave (GUERRA, 2020).

Dessa maneira, as medidas de controle e as restrições da pandemia, afetaram negativamente com forte impacto ao agronegócio (SCHNEIDER et al., 2020), causando paralização total nos embarques e desembarques de produtos, especialmente aqueles de origem internacional, devido à insegurança, prejudicando o trânsito e o suprimento de alimentos entre os países (JAIME, 2020).

Nesse cenário, a população se viu numa situação de possível desabastecimento e uma possível escassez de alimentos. Assim, em diversos países, verificou-se que rapidamente, com medo da falta de produtos alimentares e de higiene acabassem, houve uma grande onda de "compras de pânico", em que as pessoas começaram a estocar alimentos, causando assim um desabastecimento de determinados 
produtos, aumentando a demanda de alimentos básicos. Como consequência, o preço de muitos produtos aumentaram estratosfericamente, sendo que a maioria compunha a cesta básica (SIPIONI et al., 2020).

Esse aumento repentino na demanda do varejo, por conta do desabastecimento acelerado, causado pelo pânico da população, dificultou a reposição de diversos itens em muitos países. Dessa maneira, Soendergaard et al. (2020) destacam que as incertezas sobre a crise sanitária e econômica configuraram uma situação de crise global não só de saúde, mas também de segurança alimentar.

Já citada anteriormente, as medidas de restrição de comércio e circulação de pessoas, pode ter sido um dos fatores responsáveis pela elevação dos preços dos produtos, afetando a população mais carente, causando a insegurança alimentar, que consequentemente tem causado desemprego e perda da renda de muitas famílias (SIPIONI et al., 2020).

$\mathrm{O}$ agronegócio é a principal atividade que move a economia brasileira, e ocupa uma posição fundamental, que tem mantido ao longo de décadas, um balanço positivo na economia brasileira (DA SILVA et al., 2013).

Entretanto, a insegurança generalizada no mercado internacional decorrente da pandemia (OLIVEIRA, 2020), levou a impactos negativos e muitos desafios no setor brasileiro, como a queda nas exportações, queda na demanda interna, externa e aumento dos custos de produção. $O$ produtor rural se viu na condição de reaprender a produzir, com restrições e elevação de valores ao longo de toda a cadeia produtiva.

Um dos maiores desafios causado pela pandemia foi na área da saúde e no estilo de vida da população brasileira, afetando de diferentes formas, principalmente o sistema alimentar. E, diante desse cenário, a insegurança alimentar foi o que mais impactou a população mais frágil. 0 governo brasileiro, para mitigar os impactos da pandemia, apresentou uma ação relacionada à renda, como o auxílio emergencial, buscando amenizar os efeitos causados pela paralização da economia e que afetou sobremaneira a população com menor poder aquisitivo, tornando-a extremamente vulnerável (ALPINO et al., 2020).

O governo adotou ações de transferência direta de renda como forma de combater a crise econômica e alimentar vivenciada pelos trabalhadores, desempregados, microempreendedores, autônomos e informais (TOMAZELLI; FERNANDES, 2020). Segundo a Lei no 13.982, de 02 de abril de 2020, todos os setores citados anteriormente, tiveram o direito de solicitar o auxílio emergencial. Os trabalhadores, por conta do isolamento social e a desaceleração econômica, enfrentaram diversas dificuldades e, muitos perderam suas fontes de renda (ALPINO et al., 2020).

No entanto, o agronegócio, apesar da demanda de produção, as interrupções nos mercados agroalimentares, os quais afetaram a produção e disponibilidade de alimentos, ainda conseguia manter a capacidade ativa de geração de riqueza e renda, mantendo não só seu setor produtivo, mas também sustentando a economia nacional, assolada pela devastação econômica que passa (ALPINO et al., 2020).

A redução na capacidade produtiva de alimentos foi visível, sendo que em alguns setores, foi muito mais proeminente, como a dos produtores de hortaliças que, não havendo restaurantes para consumo de seus produtos que, na maioria, eram consumidos "in natura", tiveram suas atividades paralisadas por completo.

Outros setores como a produção de grãos, apesar do aumento no custo de produção, devido à escassez de insumos para o cultivo, ainda assim sofreram menos o efeito da pandemia global.

$\mathrm{E}$, diante desse cenário, o surto global do novo coronavírus poderá afetar a colheita em 2020/21, ou seja, afetando a importação dos produtos do agronegócio brasileiro, acarretando na diminuição do crescimento, logística e consumo dos produtos advindos do campo.

A crise causada pelo novo coronavírus está diretamente ligada ao sistema agroalimentar global, principalmente quando se refere à produção e ao abastecimento. Os efeitos iniciais da pandemia, foram duplos, sendo na oferta e na demanda, em consequência das medidas impostas pela OMS, interrompendo a logística das produções e no escoamento dos produtos.

As restrições causaram prejuízos aos países que são produtores, importadores e exportadores de alimentos, e o Brasil está entre esses países. Os prejuízos provocados por esta conjuntura são maiores quando a situação da população é mais vulnerável. Entretanto, cabe salientar que essa situação de vulnerabilidade já existia antes mesmo do novo coronavírus, no entanto, acentuou ainda mais no decorrer da 
pandemia. Apesar da tragédia sanitária causada pelo novo coronavírus, apontando consequências sociais graves e econômicas, com acentuação da pobreza desumana (SIPIONI et al., 2020). O agronegócio tem se recuperado, mostrando crescimento e reduzindo os impactos gerados pelo novo coronavírus.

Assim, mesmo em meio ao cenário da crise causada pelo Covid-19, o agronegócio tem apresentado resultados satisfatórios, os quais mostram amenização do impacto sofrido pela economia brasileira, que deve ser a razão da recuperação (CIRILLO, 2020).

Devido a esse impacto negativo da pandemia sobre as diferentes atividades do setor, dados do IBGE sobre o crescimento do PIB do agronegócio demonstram que este foi lento em abril e maio no ano de 2020. Mas tem havido uma sensível aceleração desde junho, e as perspectivas de crescimento anual recorde em 2021 estão tomando forma.

Nesta situação incerta, é difícil para os produtores e o agronegócio fazerem planos a curto e médio prazo para as próximas safras. Não é possível adivinhar ou profetizar o quanto a safra do ano seguinte será melhor ou pior do que a atual. Entretanto, a conjuntura vai depender do tempo de resposta do setor ao enfrentamento da pandemia.

Apesar da alta do dólar ter provocado reações positivas nas exportações de produtos brasileiros, essa realidade começou a mudar quando da necessidade de importação de insumos e dependência de maquinas e componentes que não poderiam ser fabricados no Brasil. Assim, por conta da grande dependência de insumos importados, o Brasil precisa manter um potencial produtivo que the garanta a sustentabilidade, buscando soluções e reduzindo custos e desperdícios em todo o processo produtivo.

Mais de $70 \%$ de insumos agropecuários são importados para o Brasil. E, devido à alta do dólar, os consumidores estão sentindo o impacto nos custos de produção na hora da compra dos alimentos. E o plano de colheita deve considerar todos esses aspectos que influenciam a produção, a demanda, a exportação e a importação de produtos do agronegócio.

E, diante dos apontamentos, as perspectivas macroeconômicas para 0 agronegócio são vistas com muita expectativa positiva. O IPEA aponta um crescimento de 1,5\% para o ano de 2021. A CONAB prevê aumento de
$3,1 \%$ na produção dos grãos, com destaque positivo para soja com $7,1 \%$. Porém, o arroz e o feijão, que compõem a alimentação básica de todo o brasileiro, estão com previsão de queda de 2,5\%; o algodão também terá queda de $12 \%$ e a previsão é estabilidade para o milho. Já na pecuária, a expectativa é aumento de $5,5 \%$, e para a produção de suínos, espera-se um aumento de 3,5\% (BARROS; CASTRO, 2021).

$\mathrm{O}$ agronegócio brasileiro mais uma vez se mostrou um setor forte, apresentando bons resultados em meio a um período de retração econômica, inúmeros desafios provocados pela pandemia de Covid-19. A pesar dos inúmeros impactos vivenciado pelo agronegócio, o setor se destacou, apresentando resultados positivos.

\section{CONSIDERAÇÕES FINAIS}

Os impactos econômicos decorrentes da crise do novo coronavírus, principalmente no sistema agroalimentar, afetou de forma direta e indireta o abastecimento, contribuindo para uma forte insegurança alimentar.

A conexão ampla entre os sistemas alimentares inclina-se a amplificar os efeitos da crise provocada pela pandemia, podendo oferecer riscos à população menos favorecida. As restrições no comércio de alimentos, ocorrido em vários setores (restaurantes, lanchonete, feiras, mercados entre outros), onde as restrições provocaram inúmeros prejuízos.

Estas restrições que afetam a exportação e importação de alimentos, foi mais prejudicial aos países que importam alimentos, principalmente os países que têm uma porcentagem significativa da população mais carente, no qual em sua maioria já se encontravam em uma situação alimentar precária antes do início da pandemia, e a crise provocada pelo novo coronavírus veio acentuando mais esta situação.

Essa crise está diretamente relacionada ao isolamento social. Quanto mais amplo o período de isolamento, maiores foram e podem aumentar os impactos.

Os efeitos iniciais da crise do novo coronavírus foram duplos em diversas regiões, afetando a oferta, havendo interrupções na produção e na demanda.

A queda da renda das famílias foi outro fator que trouxe novamente o medo da insegurança alimentar, com a redução da renda muitas famílias mudaram o estilo de vida e alimentação, isso tem um alto peso quando 
falamos das famílias mais carentes, onde a pandemia contribui na acentuação da pobreza e insegurança alimentar.

Os custos da crise e das paralisações são de grande magnitude, no entanto a previsões positivas para a recuperação da economia brasileira através do crescimento do agronegócio em meio a pandemia.

O Brasil é um grande fornecedor de alimentos, onde é visto como um país que pode assegurar a segurança alimentar, o país tem aumentado as exportações de alimentos, abastecendo outros países, o que significa que o Brasil tem capacidade de exportar e ainda garantir suas próprias necessidades. E O agronegócio brasileiro é visto como garantidor de segurança alimentar global e crescimento da economia brasileira.

E diante dessa crise decorrente do novo coronavírus que o mundo está vivenciado, é possível notar que vários países estão se adaptando à nova realidade, buscando vencer esse novo desafio que a natureza empregou. E o que reforça essa nova adaptação ao novo coronavírus, são os inúmeros estudos e vacinas desenvolvidas, que traz mais ânimo nessa batalha.

\section{AGRADECIMENTOS}

O presente trabalho foi realizado com apoio da Coordenação de Aperfeiçoamento de Pessoas de Nível Superior - Brasil (CAPES) e do Programa de Pós-graduação em Biodiversidade e Agroecossistemas Amazônicos da Universidade do Estado de Mato Grosso.

\section{REFERÊNCIAS}

ALPINO, T. D. M. A.; SANTOS, C. R. B.; BARROS, D. C. D.; FREITAS, C. M. D. COVID-19 e (in) segurança alimentar e nutricional: ações do Governo Federal brasileiro na pandemia frente aos desmontes orçamentários e institucionais. Cadernos de Saúde Pública, Rio de Janeiro, v. 36, n. 8, 2020. DOI. https://doi.org/10.1590/0102-311X00161320

BARCELLOS, M. L. S.; REUPKE, E. G. Coronavírus (COVID-19) - a possibilidade de tratamento compulsório de acordo com a lei no 13.979/2020. Revista da ESMESC, Santa Catarina, v. 27, n. 33, p. $141 \quad-\quad 166,2020 . \quad$ DOI. https://doi.org/10.14295/revistadaesmesc.v27i3 3.p141
DA SILVA, M. G.; CESARIO, A. V.; CAVALCANTI, I. R. Relevância do agronegócio para a economia brasileira atual. Apresentado em X ENCONTRO DE INICIAÇÃO À DOCÊNCIA, UNIVERSIDADE FEDERAL DA PARAÍBA. Recuperado de http://www. prac. ufpb. br/anais/IXEnex/iniciacao/documentos/anais/8. TRABALHO/8C CSADAMT01. pdf, 2013.

BARROS, G. S. C.; CASTRO, N. R. Perspectivas macroeconômicas para o agronegócio em 2021. Piracicaba: Esalq/USP, 2021. Disponível em: https://www.cepea.esalq.usp.br/br/opiniaocepea/perspectivas-macroeconomicas-para-oagronegocio-em-

2021.aspx\#: :text=0\%20lpea\%20trabalha\%20co m\%20uma,queda\%20de\%202\%2C5\%25. Acesso em: 04 de março de 2021.

BARROS, G. S. C.; CASTRO, N. R. CEPEA: breve retrospecto macroeconômico do agronegócio em 2020. Piracicaba: Esalq/USP, $2020 . \quad$ Disponível em: https://www.cepea.esalq.usp.br/br/releases/cep ea-breve-retrospecto-macroeconomico-doagronegocio-em-2020.aspx. Acesso em: 30 de março de 2020.

CIRILLO, B. Agronegócio cresce, reduz o tombo da economia e deve ser motor da recuperação. Portal UOL. 15 de junho de 2020. Disponível em:. Acesso em: 20 nov. 2020.

CARDOSO, V. D.; COSTA, I. C. Impactos dos primeiros meses de pandemia de covid-19 para a agricultura familiar do Pará e como a agroecologia pode apoiar a superação. https://economia.uol.com.br/noticias/redacao/2 020/06/14/agronegocio-bate-recordes-eaumenta-seu-peso-na-economia-em-meio-apandemia.htm\#: :text=Mesmo\%20 em\%20 maio\%20\%C3\%A0\%20crise,e\%20 especialistas\%20 ouvidos\%20para\%20 reportagemAmbiente: Gestão e Desenvolvimento, Roraima, v. 1, n.1, p. 40-54, 2020. DOI. https://orcid.org/0000-0002-2586$\underline{8928}$.

CNA. Confederação da Agricultura e Pecuária do Brasil. Avalia impactos do coronavírus no agronegócio. Brasília: CNA, 2020. Disponível em: https://www.cnabrasil.org.br/noticias/faepavalia-impactos-do-coronavirus-no-agronegocio. Acesso em 04 março 2021. 
CUNHA, R. C.; ESPÍNDOLA, C. J. A dinâmica geoeconômica recente da cadeia produtiva da soja no Brasil e no mundo. GeoTextos, Bahia v. 11, n. $1, \quad 2015 . \quad$ DOI. http://dx.doi.org/10.9771/1984-

5537geo.v11i1.12692.

DUARTE, P. M. COVID-19: Origem do novo coronavírus/COVID-19: Origin of the new coronavirus. Brazilian Journal of Health Review, Espirito Santos, v. 3, n. 2, p. 3585-3590, 2020. DOI. https://doi.org/10.34119/bjhrv3n2-187

Editora Globo. Economia: China detecta coronavírus em carne congelada e embalagens vindas de Brasil e outros países. Disponível em: https://oglobo.globo.com/economia/chinadetecta-coronavirus-em-carne-congeladaembalagens-vindas-de-brasil-outros-paises-124747767\#newsletterLink. Acesso em: 15 nov. 2020.

GUASQUE, B.; GUASQUE, A. A pandemia e o necessário e tempestivo retorno aos matizes keynesianos. Opinión Jurídica, Fortaleza, v. 19, n. 40, p. 259-276, $2020 . \quad$ DOI. https://doi.org/10.22395/ojum.v19n40a13.

GUERRA, H. B. Do sonho ao pesadelo: Dilemas de quem demitiu na pandemia. CASOTECA Edição Especial: Os impactos da Pandemia no Segmentos do Comércio de Bens, Serviços e Turismo, São Paulo, v. 2, n. 2, p. 1-14, 2020. Disponível em: https://scholar.google.com.br/scholar?hl=ptBR\&as_sdt $=0 \% 2 \mathrm{C} 5 \& \mathrm{q}=$ Do+sonho+ao+pesadelo\% $3 \mathrm{~A}+$ Dilemas+de+quem+demitiu+na+pandemia.+C ASOTECA+Edi\%C3\%A7\%C3\%A3o+Especial\%3A+Os +impactos+da+Pandemia+no+Segmentos+do+Co m\%C3\%A9rcio+de+Bens\%2C+Servi\%C3\%A7os+e+ Turismo+\%28ISSN\%3A+2675-4703\%29\&btnG=. Acesso em: 20 nov. 2020.

HEREDIA, B.; PALMEIRA, M.; LEITE, S. P. Sociedade e economia do "agronegócio" no Brasil. Revista brasileira de ciências sociais, São Paulo v. 25, n. 74, p. 159-176, 2010. https://doi.org/10.1590/S0102$\underline{69092010000300010}$

JAIME, P. C. Pandemia de COVID19: implicações para (in) segurança alimentar e nutricional. Ciência \& Saúde Coletiva. Rio de Janeiro, v. 25, n.
$7, \quad$ p. 2504-2504, 2020 DOl. https://doi.org/10.1590/1413-

81232020257.12852020.

LAU, H.; KHOSRAWIPOUR, V.; KOCBACH, P.; MIKOLAJCZYK, A.; SCHUBERT, J.; BANIA, J.; KHOSRAWIPOUR, T. The positive impact of lockdown in Wuhan on containing the COVID-19 outbreak in China. Journal of travel medicine, Estados Unidos, v. 27, n. 3, p. 37, 2020. DOI. https://doi.org/10.1093/jtm/taaa037.

LEIVA, G. D. C.; SATHLER, D.; ORRICO FILHO, R. D. Estrutura urbana e mobilidade populacional: implicações para o distanciamento social e disseminação da Covid-19. Revista Brasileira de Estudos de População, São Paulo, v. 37, 2020. DOI. https://doi.org/10.20947/S0102-3098a0118.

MAURI, G. D. N.; DE LIMA, J. G.; POZO, O. V. C.; DE FREITAS, R. R. Startups no agronegócio brasileiro: uma revisão sobre as potencialidades do setor. Brazilian Journal of Production Engineering-BJPE, Espírito Santo, v. 3, n. 1, p. 107-121, 2017. Disponível em: http://periodicos.ufes.br/BJPE. Acesso em: 20 nov. 2020.

NEVES, M. F.; KALAKI, R. B.; RODRIGUES, J. M.; GRAY, A. W. Planejamento estratégico e gestão de cadeias de alimentos e do agronegócio: o método ChainPlan (estrutural). Revista Brasileira de Gestão de Negócios, São Paulo, v. 21, n. 4, p. 628-646, 2019. DOI. 10.7819/rbgn.v21i4.4012.

NONNENBERG, M. J. B. China: estabilidade e crescimento econômico. Brazilian Journal of Political Economy, São Paulo, v. 30, n. 2, p. 201218, 2010. DOI. https://doi.org/10.1590/S010131572010000200002

OLIVEIRA, C. M. Insegurança Hídrica em Tempos de Covid-19. In: Valencio, N.; OLIVEIRA, C. M. (ed.). Covid-19: crises entremeadas no contexto de pandemia (antecedentes, cenários e recomendações). São Carlos: UFSCar/CPOI, 2020. p.121-134. Disponível em: https://scholar.google.com.br/scholar?hl=ptBR\&as_sdt $=0 \% 2 C 5 \& q=O l i v e i r a \% 2 C+C .+M .+\% 282$ 020\%29.+Inseguran\%C3\%A7a+H\%C3\%ADdrica+e m+Tempos+de+Covid-

19.+In\%3A+Valencio\%2C+N.+e+Oliveira\%2C+C.+ M.+\%28Ed.\%29\%2C+Covid-

$19 \% 3 \mathrm{~A}+$ crises+entremeadas+no+contexto+de++p andemia++\%28antecedentes\%2C++cen\%C3\%A1ri 
os++e++recomenda\%C3\%A7\%C3\%B5es\%29++\%2

8pp.121-

134\%29.+S\%C3\%A3o+Carlos\%3A+UFSCar\%2FCPO I.\&btnG=. Acesso em: 5 jul. 2021.

OMS. Organização Mundial da Saúde. Covid-19: Global data. 2020. Disponível em: https://covid19.who.int. Acesso em: 16 nov. 2020.

QUN LI, et al. Early Transmission Dynamics in Wuhan, China, of Novel Coronavirus-Infected Pneumonia. New England Journal of medicine, Massachusetts, v. 38, n. 13, p. 1-9, 2020. DOI: https://doi.org/10.1056/NEJMoa2001316

SCHNEIDER, S.; CASSOL, A.; LEONARDI, A.; MARINHO, M. D. M. Os efeitos da pandemia da Covid-19 sobre o agronegócio e a alimentação. Estudos Avançados, São Paulo, v. 34, n. 100, p. 167-188, 2020. DOI. https://doi.org/10.1590/s01034014.2020.34100.011

SIPIONI, M. E.; RIQUIERI, M. R. L.; BARBOSA, J. P. M.; BISCOTTO, D. B.; SARTI, T. D.; ANDRADE, M. A. C. Máscaras cobrem o rosto, a fome desmascara o resto: COVID-19 e o enfrentamento à fome no Brasil. Scientific Electronic Library Online, São Paulo, p. 1- 21, 2020. DOI. https://doi.org/10.1590/SciELOPreprints.660

SOENDERGAARD, N.; GILIO, L.; DE SÁ, C. D.; JANK, M. S. Impactos da covid-19 no agronegócio e o papel do Brasil. Insper - Centro do Agronegócio Global. n. 2, jun. 2020. Disponível em: https://www.insper.edu.br/wpcontent/uploads/2020/06/impactos-da-covid-19no-agronegocio-e-o-papel-do-brasil-vf-a.pdf.

Acesso em: 30 mar. 2021.

TALIARINE, A. B.; RAMOS, D. J. ; FAVORETTO, M. J. R. A. Importância da gestão no agronegócio brasileiro. Revista Perspectiva em Gestão, Educação \& Tecnologia, São Paulo, v. 4, n. 8, p. 6, $2015 . \quad$ Disponível em: https://scholar.google.com.br/scholar?hl=ptBR\&as_sdt=0\%2C5\&q=.+Import\%C3\%A2ncia+da+ gest $\%$ C3\%A3o+no+agroneg\%C3\%B3cio+brasileiro \&btnG=.

TOMAZELLI, I.; FERNANDES, A. Transferência de renda é adotada em 30 países. UOL Economia. 2020. Disponível em: conteudo/2020/03/24/transferencia-de-renda-eadotada-em-30-paises.htm. Acesso em: 24 mar. de 2020 .

ZHU, et al. A novel coronavirus from patients with pneumonia in China. The new england journal of medicine, p. 382, fev. 2019. Disponível em: https://www.nejm.org/doi/full/10.1056/nejmoa2 001017. Acesso em: 5 jul. 2021.

https://economia.uol.com.br/noticias/estadao- 